\title{
ROBOTIC FLIGHT FOLLOWER SYSTEM FOR HIGH SPEED RECORDING
}

\author{
Daniil Klimov, Yury Poduraev \\ Moscow State University of Technology “Stankin”, Vadkovskiy per. 1, Moscow 127994, Russia
}

\begin{abstract}
High-speed filming is becoming an important tool for scientific, industrial and military research. Some studies require the ability to extract data from fast ballistic events, such as projectile in-flight behavior. Due to very high speeds, in flight follower systems the camera is kept stationary and the image of the moving object is relayed into the camera via a fast panning mirror. This paper proposes the use of an industrial manipulator for the construction of a system for high-speed recording of dynamic objects. Unlike some commercial mechatronic counterparts the robotic system will allow shooting objects moving not only along linear, but also more complex trajectories, in a wide range of speeds. The original layout using two mirrors will allow winning in speed, volume of the work area, and compensating distortion of the object's orientation in the frame.
\end{abstract}

Keywords: robot; high speed; camera; flight follower; ballistic; in-flight behavior;
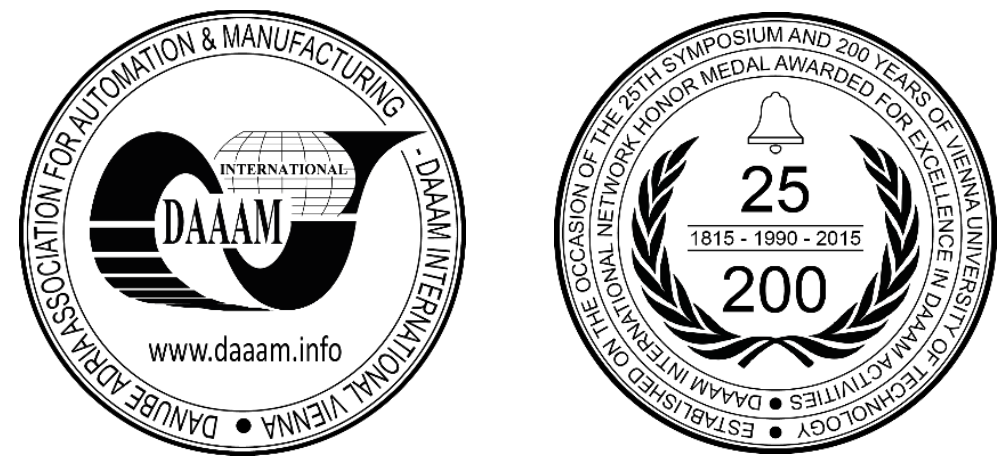

This Publication has to be referred as: Klimov, D[aniil] \& Poduraev, Y[ury] (2016). Robotic Flight Follower System for High Speed Recording, Proceedings of the 26th DAAAM International Symposium, pp.0720-0726, B. Katalinic (Ed.), Published by DAAAM International, ISBN 978-3-902734-07-5, ISSN 1726-9679, Vienna, Austria DOI: $10.2507 / 26$ th.daaam.proceedings.100 


\section{Introduction}

In many fields of knowledge there are tasks that require filming behaviour of a dynamic object using high-speed cameras. In particular in military and sports science, but also in the film and television industry. For example in order to correctly identify the failure modes of projectiles, it is often necessary to capture its behaviour on a significant portion of the flight path. Another example would be studying ball rotation during its flight. Using a series of cameras to achieve this objective is ineffective, since the system will be deprived of universality and would be unduly expensive. The classical approach, first used in 1962 by the division of the British Ministry of Defence RARDE (Royal Armament Research and Development Establishment), implies the use of a rotating mirror [1]. It gives a distinct advantage - the angular velocity required to track the object is reduced by half. This is due to the fundamental law of reflection of light - angle of incidence on a reflecting surface equals the angle of reflection. Accordingly, in order to rotate the beam by the angle $\alpha$, the normal vector of the mirror must be displaced by an angle equal to $\alpha / 2$ (see Fig. 1).

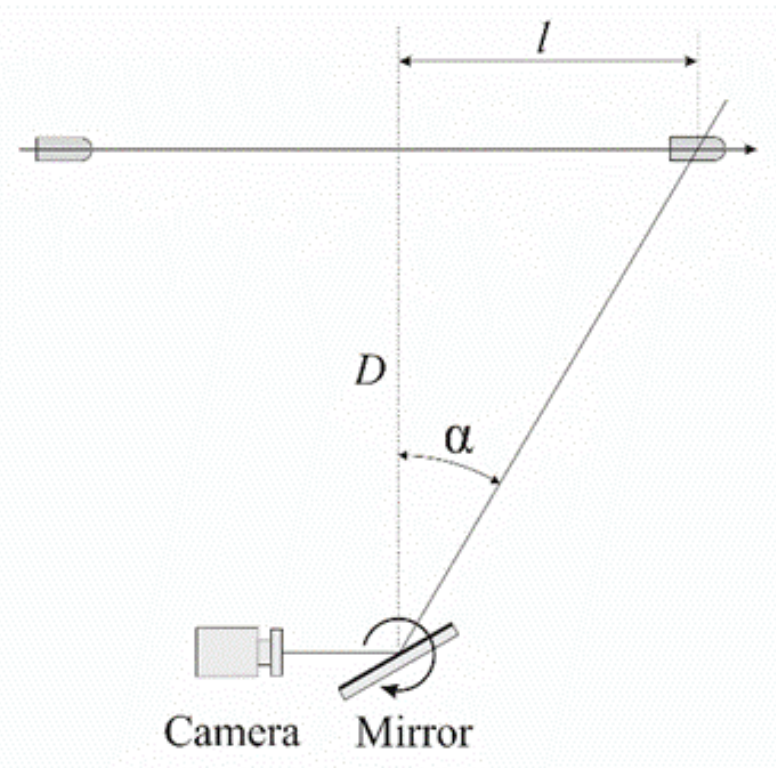

Fig. 1. The scheme of the approach, using one rotating mirror.

An additional advantage of this method is the fact, that the high-speed camera is stationary, and does not require the ability to withstand high acceleration force. Also, object tracking can increase exposure time, which in turn reduces requirements for lighting equipment and improves quality of the resulting footage. In the early sixties, mirrors were mounted on large galvanometer coils and driven with a series of increasing pulses from storage capacitors. Modern industrial systems, such as Tracker2 Flight Follower of the British company Specialized Imaging [2], use servos. A similar method was used by Japanese scientists from the University of Tokyo, applying two mirrors for pan and tilt, respectively. Their system allows tracking an object in real-time, processing video signal at a frequency of $1 \mathrm{KHz}$. However, the angle of view of the system does not exceed 40 degrees [3]. This principle, using fast rotating mirrors is also applied in laser scanning [4] and material processing [5], where there is a need for reaching high scanning speeds. More complex systems, with multiple mirrors are used for stereo vision in micro-part assembly [6].

In this paper, we propose an original two-mirror optical layout and its implementation based on an industrial manipulator. It allows winning in volume of the working area, speed, and, optionally, will be able to compensate orientation distortion of the object in the frame. Unlike some industrial mechatronic counterparts, the robotic system will allow filming objects moving not only along linear, but also more complex trajectories, at a wide range of speeds.

\section{Design}

\subsection{Optical layout}

The scheme includes two mirrors (see Fig.2 a, b). The secondary mirror is placed parallel to the XZ plane at the point $\mathrm{O}^{\prime}(0,0, \mathrm{~lm})$, and has one rotational degree of freedom, parallel to the $\mathrm{z}$ axis. The primary mirror can be moved along a circular path around the center of the secondary mirror at an angle $\gamma$. It has two rotational degrees of freedom - $\alpha$ (pan) and $\beta$ (tilt). Its path is displaced along the $\mathrm{z}$ axis in the $\operatorname{lm} \sin (\psi)$. The optical axis of the camera is rotated around the point $\mathrm{O}^{\prime}$ by the angle $\psi$. 

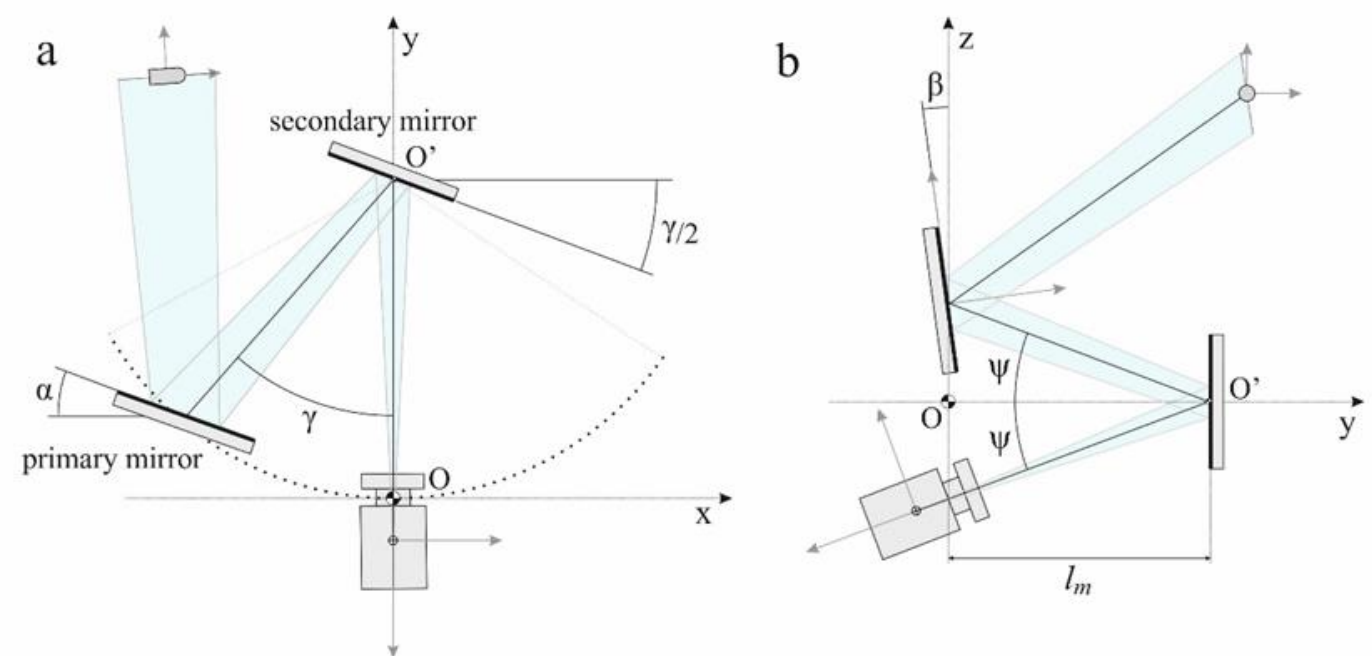

Fig. 2. The proposed optical layout, using two mirrors (a) top view; (b) side view.

Both mirrors should be oriented in space in such a way, that the light reflected from the object of shooting is relayed into the camera at any given moment.

\subsection{Inverse kinematics}

The cartesian coordinates of the primary mirror is defined as:

$$
x_{\text {mir }}=l_{m} \cdot \sin \gamma ;, y_{m i r}=l_{m}-l_{m} \cdot \cos \gamma ; z_{m i r}=-l_{m} \cdot \sin \psi
$$

The direction vector at the object being filmed $v$ :

$$
\bar{v}=\left[\begin{array}{c}
\left(x_{o b j}-x_{m i r}\right) \cdot \cos \gamma-\left(y_{o b j}-y_{m i r}\right) \cdot \sin \gamma \\
\left(x_{o b j}-x_{m i r}\right) \cdot \sin (-\gamma)+\left(y_{o b j}-y_{m i r}\right) \cdot \cos \gamma \\
z_{o b j}-z_{m i r}
\end{array}\right]
$$

where $x_{o b j,} y_{o b j}, z_{o b j}-$ the coordinates of the object.

The vector of the optical axis of the camera $c$ :

$$
\bar{c}=\left[\begin{array}{c}
0 \\
-\cos \psi \cdot l_{m} \\
-\sin \psi \cdot l_{m}
\end{array}\right]
$$

The vector of the normal to the surface of the primary mirror $\boldsymbol{m}$ is the sum of normalized vectors:

$$
\bar{m}=\hat{v}+\hat{c}
$$

The rotational degrees of freedom of the primary mirror:

$$
\left\{\begin{array}{l}
\alpha=\gamma-\operatorname{arctg}\left(\frac{m_{x}}{m_{y}}\right)+\psi \\
\beta=90-\operatorname{arctg}\left(\frac{m_{z}}{\sqrt{m_{x}^{2}+m_{y}^{2}}}\right)
\end{array}\right.
$$

The angles $\alpha$ and $\beta$ are limited by design parameters and must belong to a certain range. 


\subsection{Work zone}

Let's consider the function of visibility by direction $V(\boldsymbol{\theta}, \boldsymbol{\varphi}, \boldsymbol{\gamma})$ defined as:

$$
V(\theta, \varphi, \gamma)=\left\{\begin{array}{l}
1, \alpha \in\left[\alpha_{\min }, \alpha_{\text {max }}\right] \wedge \beta \in\left[\beta_{\text {min }}, \beta_{\text {max }}\right] \\
0, \text { otherwise }
\end{array}\right.
$$

where $\alpha$ and $\beta$ are calculated according to the formula (5).

In a spherical coordinate system, a measure of accessibility $\boldsymbol{A}(\boldsymbol{\theta}, \boldsymbol{\varphi})$ by direction is proposed to determine by the percentage of the $\gamma$ angle (of total range) in which the visibility function $\boldsymbol{V}(\boldsymbol{\theta}, \boldsymbol{\varphi}, \boldsymbol{\gamma})$ gives a non-zero result. This approach can be expressed as follows:

$$
A(\theta, \varphi)=\frac{\int_{\gamma_{\min }}^{\gamma_{\max }} V(\theta, \varphi, \gamma) d \gamma}{\gamma_{\max }-\gamma_{\min }}
$$

In order for the system to be able to lock on any point on the hemisphere around the origin, under the condition that the angle between the primary and secondary mirror does not exceed 90 degrees, the angle $\gamma$ should be in the minimum range of $+/$ - 60 degrees.

\subsection{Mirror sizes}

The minimum required size of the primary and secondary mirrors are determined by the minimum focal length of the lens and the physical size of the digital matrix(or film) used for shooting. For the secondary mirror there are the following relationships:

$$
\begin{aligned}
& \text { Width }_{\text {SecondMir }}=\frac{\left(\frac{L / \cos (\psi)}{F_{\text {min }}}-1\right) \cdot \text { Width }_{\text {matrix }}}{\cos \left(\gamma_{\max } / 2\right)} ; \\
& \text { Height }_{\text {SecondMir }}=\frac{\text { Width }_{\text {SecondMir }}}{\text { Aspect Ratio } \cdot \cos (\psi)} ;
\end{aligned}
$$

For the primary mirror there are the following relationships:

$$
\begin{aligned}
& \text { Width }_{\text {PrimaryMir }}=\frac{\left(\frac{2 \cdot L / \cos (\psi)}{F_{\min }}-1\right) \cdot \text { Width }_{\text {matrix }}}{\cos \left(\alpha-\frac{\gamma_{\max }}{2}\right)} ; \\
& \text { Height }_{\text {PrimaryMir }}=\frac{\text { Width }_{\text {PrimaryMir }}}{\text { Aspect Ratio } \cdot \cos \left(\psi+\beta_{\max }\right)}
\end{aligned}
$$

where $\mathrm{F}$ is the focal length of the camera expressed in $\mathrm{mm}$.

\subsection{Speed parameters}

One advantage of this optical scheme is the possibility to use the angle $\gamma$ to reduce the maximum angular speed of the primary mirror. Let us regard a motion path of an object that is symmetrical with respect to the YZ plane (see. Fig. $3 \mathrm{a}$ ). When the angle $\gamma$ is fixed, the scheme is converted to a single-mirror. Figure $3 \mathrm{~b}$ shows the blue tinted family of curves which represents $\alpha$ angular velocities at various fixed $\gamma$ angles. By changing the angle $\gamma$ while tracking the object, you can reduce the maximum $\alpha$ angular velocity. The red tinted family of curves shows the angular velocity $\alpha$, changing over a range of angle $\gamma$. 

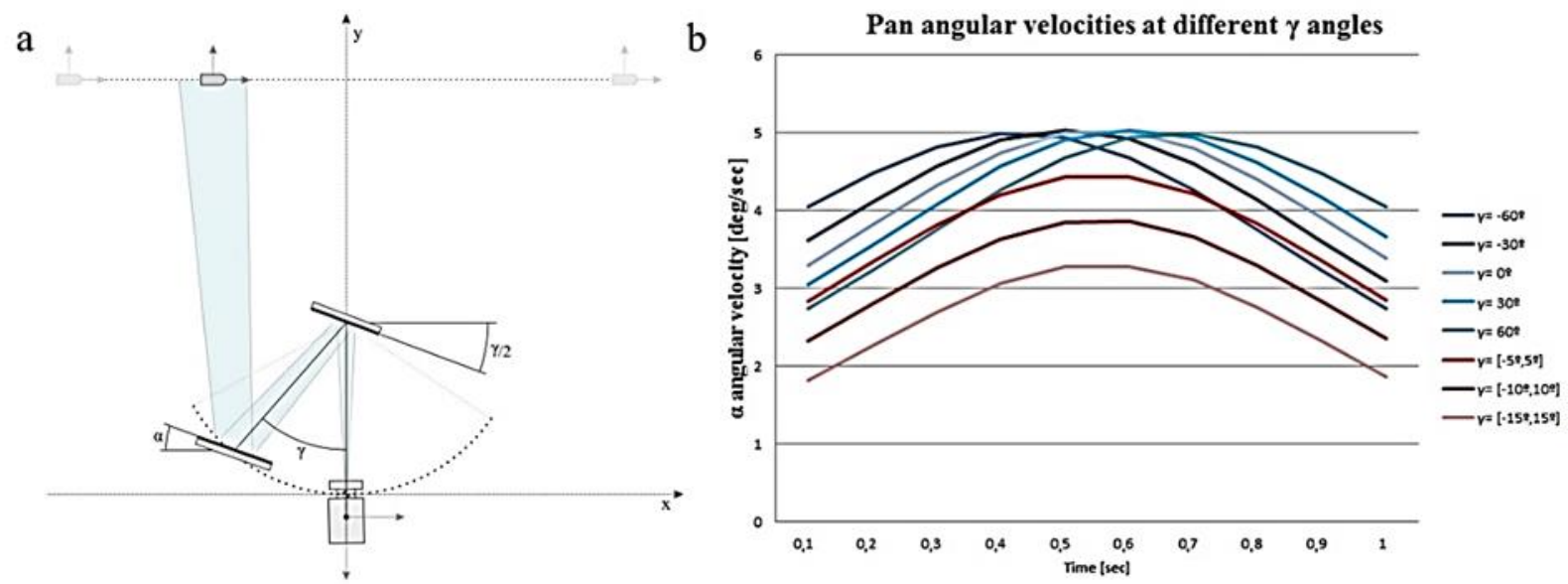

Fig. 3. (a) Top view of the layout with symmetrical trajectory; (b) Pan angular velocities at different $\gamma$ angles.

\subsection{Compensation of distortion of the object's orientation}

The nonparallelism of the primary and secondary mirrors enters distortion in the orientation of the object in the frame. Taking advantage of the fact that the system allows to view the same point in space at different $\gamma$ angles, the distortion can be compensated by adjusting the angle during filming. In this optical system the effect is more noticeable for larger angles of tilt. The value of this effect seems insignificant, since such compensation can be performed at the post-processing stage.

\section{Prototype and experiments}

\subsection{Prototype}

Due mirrors mounted both on the flange of the robot, as on an extra axis, the robotic system allows shooting fast moving objects in the field of view of up to $360^{\circ}$ in conditions of a stationary speed camera, which, in turn, allows the use of any filming equipment without taking into account its weight and dimensions. Our prototype (see Fig.4) of the robotic system is based on an industrial robot arm ABB IRB 140 with handling capacity of $6 \mathrm{~kg}$, working area radius of 0.81 meters and the maximum speed of $2.5 \mathrm{~m} / \mathrm{s}$ [4].

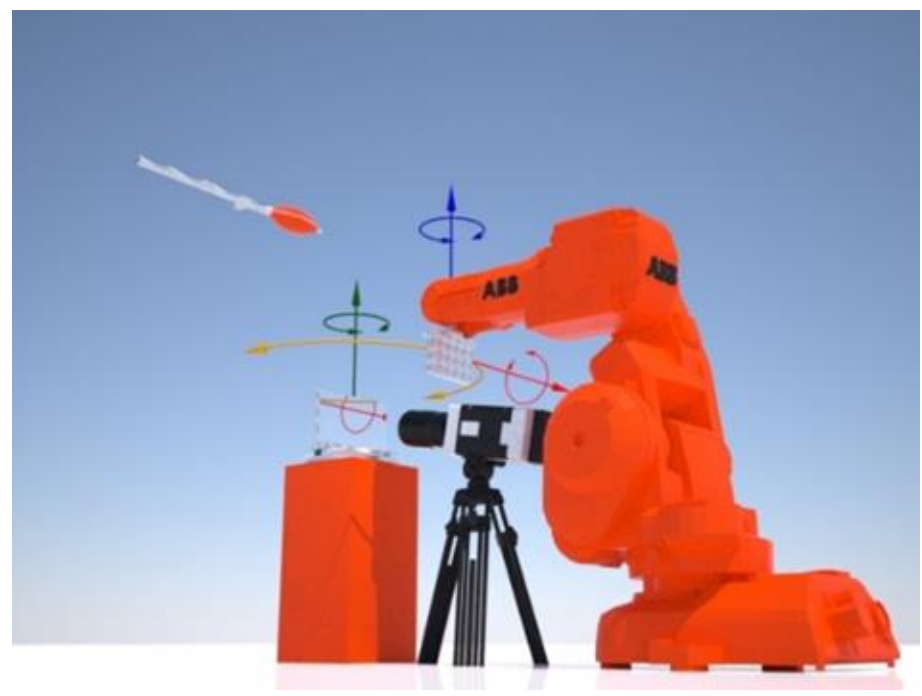

Fig. 4. Computer model of the prototype

The primary mirror is mounted on the flange of the manipulator so that the rotation axis $Z(\alpha)$ coincides with the axis of the sixth link. The secondary mirror to an additional seventh axis and is also driven by the robot controller. The industrial robot used in the prototype is not the most suitable for these tasks. Based on the requirements of speed and acceleration, better suited would be the modern ABB IRB 1200 or Kuka KR6 Agilus, having maximum speed characteristics, in particular the maximum speed of the sixth axis [5]. 


\subsection{Synchronization}

Since currently a dynamic tracking of the object is not yet implemented, the trajectory and the corresponding angles are calculated and set in advance. In this regard, there arises the demand for synchronous start of object and mirror movement. To solve this problem the system is equipped with a multi-channel synchronization device designed for this purpose in our previous work [6]. Its control system allows running up to five different processes and setting delays independently for each process, with an accuracy of $1 \mathrm{~ms}$.

\subsection{Experiment}

As an experiment filming of an arrow from a toy gun with a muzzle velocity of $18 \mathrm{~m} / \mathrm{s}$ was carried out. The object was placed at a distance of 2 meters from the robotic system. The camera Casio EX-F1 with the frequency of $600 \mathrm{fps}$ was used. The synchronization device provided simultaneous launch of a shot and the movement of the mirror. Figure 5 shows a consistent flight of the arrow on a segment of 1 meter. Figure 5a shows the initial phase before the shot. On figure $5 \mathrm{~b}$ the time of departure from the barrel is visible. The last figure (5c) shows the target's flight at a distance of 1 meter from the beginning of the movement.

a

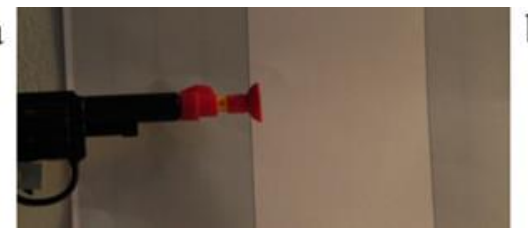

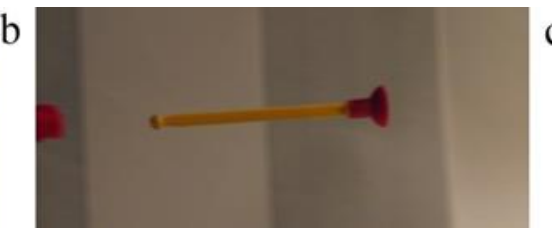

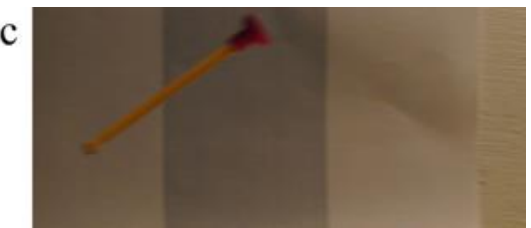

Fig. 5. (a) Initial phase before the shot; (b) Moment of departure from the barrel; (c) Flight at a distance of 1 meter;

\subsection{Visualization}

The robotic system also offers a simulation software package that allows visualizing the planned process of shooting. It is made for quick setup of the object's movement trajectory with the required speed parameters. The software package is designed as a plug-in for 3D Studio Max and Maxwell renderer (see Fig. 6a). It gives an opportunity to render photorealistic images considering parameters such as illuminance, focal length, film ISO, shutter speed and aperture [7]. This saves time during real shooting, allows choosing camera, lens and lighting equipment in advance to eliminate the possibility of occurrence of such undesirable effects like motion blur. Figure 6(b) shows an image of the virtual experiment, obtained by the simulation of the system.
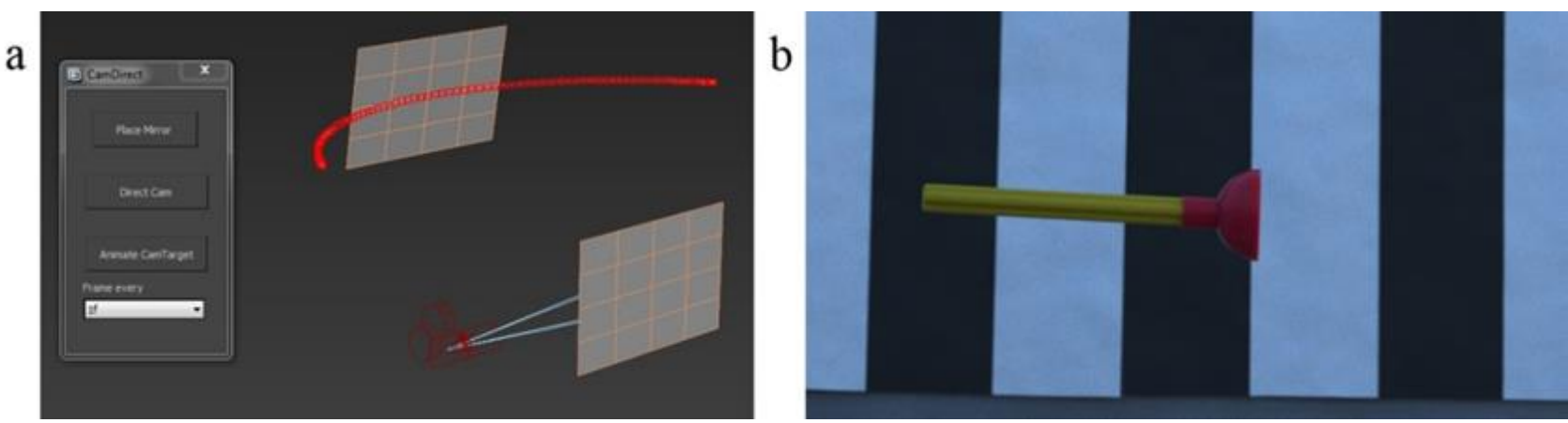

Fig. 6. (a) 3D Max plugin layout; (b) Render of the virtual experiment;

\section{Conclusion}

The goal of our work was to design a robotic system, capable of recording the behavior of rapid moving objects. The build prototype can be applied both in the film industry, and military or sport research. The proposed optical layout allows reducing angular velocities of the system primary mirror during tracking, and can be used not only for filming but also for projecting images on dynamic objects, wherein the camera is replaced by a projector [8]. It should also be noted that the above described optical layout can be implemented in a form of a mechatronic module. However, this approach would complicate the calibration, since it shall require the system to adjust the circular path of the primary mirror. In a system based on the industrial manipulator, this is not a problem.

At this point, the trajectory parameters must be known and defined in advance, which imposes requirement for repeatability of the shooting process. In perspective the system will be able to track objects in real time using additional hardware. It is planned to continue experimenting with faster objects and more complex trajectories. 


\section{References}

[1] Fuller P. W. W. Some highlights in the history of high-speed photography and photonics as applied to ballistics //High-Pressure Shock Compression of Solids VIII. - Springer Berlin Heidelberg, 2005. - C. $251-298$.

[2] Specialized Imaging - Tracker2, URL: http://specialised-imaging.com/products/tracker2-award-winning-flightfollower-system [2015-07-03]

[3] Okumura, K.; Oku, H.; Ishikawa, M., "High-speed gaze controller for millisecond-order pan/tilt camera," Robotics and Automation (ICRA), 2011 IEEE International Conference on, vol., no., pp.6186,6191, 9-13 May 2011.

[4] P. Hong-Seok, S. Chintal, Development of High Speed and High Accuracy 3D Dental Intra Oral Scanner, in: Annals of DAAAM for 2014 \& Proceedings of the 25th International DAAAM Symposium, ISSN 1877-7058, ISBN 9783-901509-97-1, Editor B[ranko] Katalinic, Published by DAAAM International, Vienna, Austria, 2014, pp. 11741181.

[5] J. Bliedtner, A. Barz, K. Hecht, A.-M. Schwager, Investigations on Laser Forming of Flat Glasses, in: Annals of DAAAM for 2014 \& Proceedings of the 25th International DAAAM Symposium, ISSN 1877-7058, ISBN 978-3901509-97-1, Editor B[ranko] Katalinic, Published by DAAAM International, Vienna, Austria, 2014, pp. 314-320.

[6] Dukyoung Lee, Min Young Kim, Hyungsuck Cho, Path planning for micro-part assembly by using active stereo vision with a rotational mirror, Sensors and Actuators A: Physical, Volume 193, 15 April 2013, Pages 201-212, ISSN 0924-4247.

[7] ABB industrial robot catalogue, URL: http://new.abb.com/products/robotics/robot-selector [2015-06-28].

[8] Kuka Agilus data sheet, URL: http://www.kukarobotics.com/en/products/industrial_robots/small_robots/kr6_r900_sixx/ [2015-06-28].

[9] D. Klimov, Y. Poduraev, Design and Experimental Testing of a Robotic System for High Speed Recording, in: Annals of DAAAM for 2014 \& Proceedings of the 25th International DAAAM Symposium, ISSN 1877-7058, ISBN 978-3-901509-97-1, Editor B[ranko] Katalinic, Published by DAAAM International, Vienna, Austria, 2014, pp. 1332-1337.

[10] Maxwell Renderer, URL: http://www.maxwellrender.com/ [2015-06-28].

[11] Okumura, K.; Oku, H.; Ishikawa, M., "Active projection AR using high-speed optical axis control and appearance estimation algorithm," Multimedia and Expo (ICME), 2013 IEEE International Conference on , vol., no., pp.1,6, 1519 July 2013. 